\title{
Optical Activity Measurements by Interferometry-Diffraction Technique: Test with BSO Crystals
}

\author{
Jorge-Enrique Rueda-P*, Dudbil-O Pabon-R, Pedro-E Rueda-P \\ Modern Optics Group, Universidad de Pamplona, Pamplona-Colombia, C.P.543050
}

Received May 01, 2014; accepted June 10, 2014; published June 30, 2014

\begin{abstract}
To measure the optical rotation of a material, we propose a technique which involves a Mach-Zehnder interferometer and an optical Fourier processor $2 \mathrm{~F}$. The method uses the contrast dependence of interference fringes with the angle between the polarization planes of two interferometer beams. The technique allows for following the dynamic variations of optical activity, directly observing the changes in the intensity of diffraction orders in the exit plane of the Fourier processor $2 \mathrm{~F}$. We present the results.
\end{abstract}

The rotation phenomenon of the plane of linearly polarized light, called optical rotation, rotation power, circular birefringence or optical activity was first measured in quartz and chiral organic liquids, in the early nineteenth century [1]. Different techniques have been proposed to study this optical phenomenon [1-13]. As an interferometry technique have been reported vibrational circular dichroism measurements by optical subtraction FT-IR spectrometry [2].

In this work, we present an interferometry technique to measure the optical rotation. The proposed technique consists of a Mach-Zehnder interferometer coupled with an optical Fourier processor 2F. Three samples of photorefractive BSO crystals were used in tests [7-13]. These crystals exhibit Biot dispersion. The merit figure was found analytically, relating optical activity of the sample with intensity variation of diffraction orders of the interference pattern. Figure 1 describes the characteristics of the implemented optical arrangement.

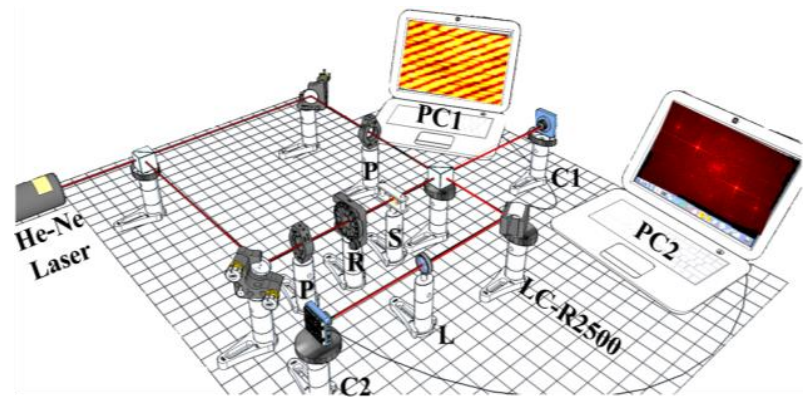

Fig. 1. Experimental arrangement: Mach-Zehnder interferometer and 2F diffraction setup. Linear polarizers $(\mathrm{P})$, half-wave retarder $(\mathrm{R})$, sample (S), CCD Moticam2300 (C1), CCD Motic352 (C2), lens (L) of 30cm focal length, Spatial Light Modulator (LC-R2500), He-Ne Laser (5mW,

${ }^{*}$ E-mail: jorgeenriquerueda@gmail.com 633nm). C1-PC1 registers interference pattern and C2-PC2 registers diffraction pattern of the interference patter.

For analytical simplicity, we assume the following scheme for interferometer (Fig. 2), where the optical fields $\tilde{E}_{1}$ and $\tilde{E}_{2}$ are linearly polarized, and their polarization planes form an angle $\varphi$ between them.

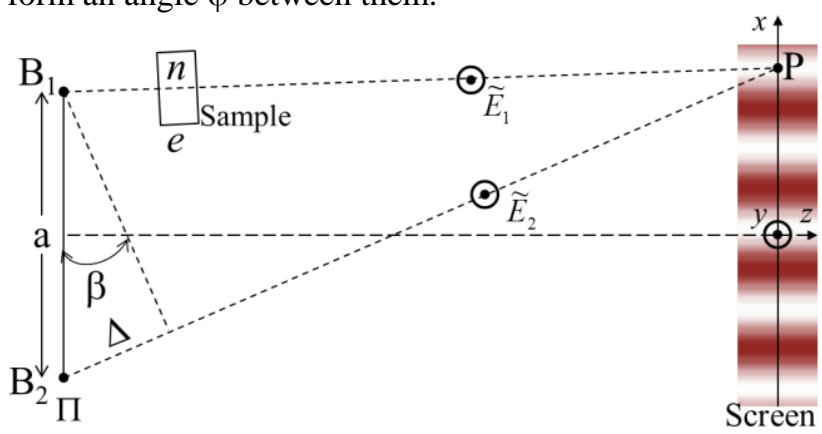

Fig. 2. Interference scheme, where $\mathrm{n}$ is the refractive index and e is the sample thickness, $a$ is the separation between the sources $B_{1}$ and $B_{2}$.

$\mathrm{B}_{1} \mathrm{P}$ and $\mathrm{B}_{2} \mathrm{P}$ are the sample and the reference beam, respectively.

The optical paths of each beam, between the distances $\overline{B_{1} P}$ and $\overline{B_{2} P}$, are:

$\Delta_{1}=n e+\left(R_{1}-e\right)=R_{1}+(n-1) e ; \Delta_{2}=R_{2}$,

where, $\mathrm{R}_{1}$ and $\mathrm{R}_{2}$ are the optical paths for each beam in free space. The optical path difference between the two beams to the point $\mathrm{P}$ on the viewing screen is:

$\Delta=\Delta_{f}-(n-1) e$,

where $\Delta_{f}$ is the optical path difference without the sample. The rotary power of the sample is defined as $\rho=\theta / \mathrm{e}$, where $\theta$ is the angular deviation of the polarization plane of the beam $B_{1}$, after passing through the sample. The optical field at the screen satisfies the relationship:

$\tilde{E}=\tilde{E}_{1} \exp \left(\mathrm{j} k \Delta_{1}\right)+\tilde{E}_{2} \exp \left(\mathrm{j} k \Delta_{2}\right)$.

Interest being the field intensity, then:

$$
\begin{aligned}
I & =I_{1}+I_{2}+\left|\tilde{E}_{1}\right|\left|\tilde{E}_{2}\right| \cos (\phi \pm \theta) \exp \left(\mathrm{j} k\left[\Delta_{1}-\Delta_{2}\right]\right)+ \\
& +\left|\tilde{E}_{2}\right|\left|\tilde{E}_{1}\right| \cos (\phi \pm \theta) \exp \left(\mathrm{j} k\left[\Delta_{1}-\Delta_{2}\right]\right),
\end{aligned}
$$

being $(+\theta)$ if the sample has the right optical activity, and $(-\theta)$ if the sample has the left optical activity. If we relate the Eq. (4) with the parameters of the optical setup, then:

$I=I_{1}+I_{2}+2 \sqrt{I_{1} I_{2}} \cos (\phi \pm \theta) \cos \left(\frac{2 \pi a}{\lambda \ell} x-k(n-1) e\right)$. 
If we consider an amplitude division interferometer, then $I_{\max }=I_{1}+I_{2}$, and we can rewrite Eq. (5) to obtain:

$I=I_{\max }\left(1+\frac{2 \sqrt{I_{1} I_{2}} \cos (\phi \pm \theta)}{I_{\max }} \cdot \cos \left(\frac{2 \pi a}{\lambda \ell} x-(n-1) \frac{2 \pi}{\lambda} e\right)\right)$,

where $m=\frac{2 \sqrt{I_{1} I_{2}} \cos (\phi \pm \theta)}{I_{1}+I_{2}}$ is the modulation of the interference pattern, $K=\frac{2 \pi a}{\lambda \ell}$ is the spatial frequency of the fringe pattern along the direction $x$, and $\Phi=(n-1) \frac{2 \pi e}{\lambda}$ is the lateral shift of the fringes due to the optical path of the sample. $\ell$ is the distance between the $\Pi$ plane and the observation screen. Then Eq. (6) can be written as:

$I=I_{\text {max }}(1+m \cdot \cos [K x-\Phi])$.

The optical activity of the sample affects the modulation of the fringes, thus measuring $m$ enables to determine the optical rotation. However, this method requires sophisticated software techniques if we want to get a result with a low error rate. Essentially, the complexity is due to the speckle noise.

We record the fringe pattern [Eq. (7)] in a liquid crystal spatial modulator LC-R2500, and this is diffracted through the optical processor $2 \mathrm{~F}$ (Fig. 1). We assume that the fringes are distributed along the $\mathrm{L}_{\mathrm{x}}$ direction, and likewise in a rectangle of $\mathrm{L}_{\mathrm{x}} \mathrm{L}_{\mathrm{y}}$ area in the LC-R2500 [14]. We also consider a monochromatic plane wave illumination, and unit amplitude. Then the diffracted field registered at the focal plane of the lens is F:

$$
U\left(K_{x}, K_{y}\right) \approx \operatorname{TF}\{\operatorname{Rect}(x, y)\} * \operatorname{TF}\left\{I\left(K_{x}, K_{y}\right)\right\},
$$

where $K_{x}=\frac{2 \pi}{\lambda F}, K_{y}=\frac{2 \pi}{\lambda F} y, \mathrm{~F}$ is the focal length of the lens L, and:

$\operatorname{Rect}(x, y)=\left\{\begin{array}{ccc}1 & \text { if } & -\left(\frac{\mathrm{L}_{x}}{2}, \frac{\mathrm{L}_{y}}{2}\right) \leq(x, y) \leq\left(\frac{\mathrm{L}_{x}}{2}, \frac{\mathrm{L}_{y}}{2}\right), \\ 0 & \text { outside. }\end{array}\right.$

Equation (10) is obtained from Eqs. (8) and (9), defining the profile of the diffracted field intensity of the direction $x$ :

$$
\begin{aligned}
& I_{U}\left(K_{x}\right)=\left(L_{x} I_{\max }\right)^{2} \cdot \operatorname{sinc}^{2}\left(\frac{L_{x}}{2} K_{x}\right)+ \\
& +\left(L_{x} I_{\max } \frac{m}{2}\right)^{2} \cdot \operatorname{sinc}^{2}\left(\frac{L_{x}}{2}\left(K_{x}-K\right)\right)+\cdot \\
& \ldots+(\text { other terms }) .
\end{aligned}
$$

From Eq. (10) we conclude that:

1) The central energy term (DC) does not change with the rotary power of the sample (only the absorption of the sample can affect this term).
2) The energy of all higher harmonics varies due to the rotatory power of the sample. Then, taking one of the higher order terms in Eq. (10), we can define the merit figure for optical rotation of the sample. Thus, if we consider, for example, the maximum intensity of the harmonic \pm 1 , we obtain the following figure of merit:

$I_{ \pm(\text {max })}=\left(L_{x} I_{\max } \frac{m}{2}\right)^{2}=L_{x}^{2} I_{1} I_{2} \cos ^{2}(\phi \pm \theta)$.

Figure 3 shows images obtained in our experiment with CCD cameras $\mathrm{C} 1$ and $\mathrm{C} 2$.

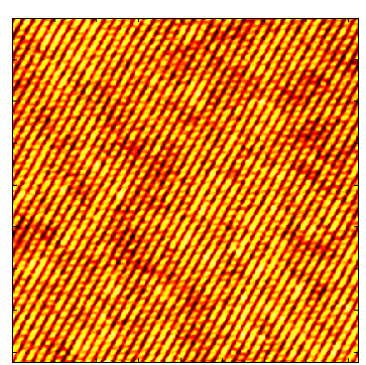

(a) Interference pattern

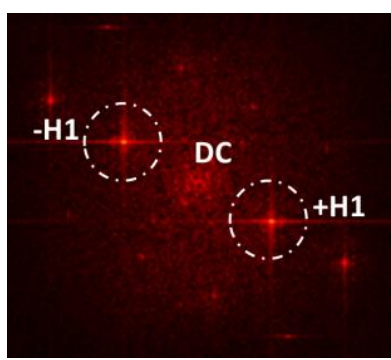

(b) Diffraction pattern of (a).
Fig. 3. Interference and diffraction patterns characteristics of the experiment mean arrangement of Fig. $1 \pm \mathrm{H} 1$ first two harmonics of diffraction.

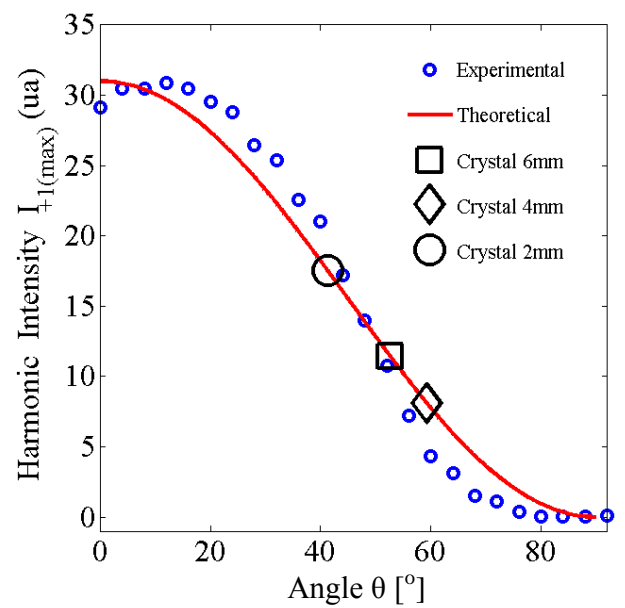

Fig. 4. Experimental results. The symbols $\square \diamond \bigcirc$ on the theoretical curve are the experimental points obtained for each BSO sample with $\varphi=0^{\circ}$. With this value the measured intensity for each sample as well as

Eq. (12) and respective values of optical rotation were determined.

Table 1. Experimental results of measuring the optical rotation of three crystals of BSO used to test the technique. IDT: InterferometryDiffraction Technique. PT: Polarimeter Technique.

\begin{tabular}{ccc}
\hline Crystal thickness $(\mathrm{mm})$ & IDT & PT \\
$\rho(\% / \mathrm{mm})$ & $\rho(\% / \mathrm{mm})$ \\
\hline 2 & 21.0058 & 21.60 \\
\hline 4 & 14.9295 & 14.40 \\
\hline 6 & 8.8451 & 7.90 \\
\hline
\end{tabular}


Different modes of operation of the technique may arise. The results presented were obtained by the following operations: first we find the experimental reference curve $I_{+1(\max )}$ without the presence of the sample (Fig. 4). Since $\varphi=0^{\circ}$ to $90^{\circ}$ the plane of polarization of the beam $B_{1}$ was rotated using the retarder $R$. Thus, by fitting the experimental data (Fig. 4), we find the reference curve given by:

$$
I_{+1(\max )}=31.7175 \cdot \cos ^{2}(\rho e) \text {. }
$$

To measure the optical rotation of each sample, first maximum modulation $\left(\varphi=0^{\circ}\right)$ was adjusted, the sample was introduced, and the $I_{+1(\max )}$ intensity was recorded by camera 2, and with this intensity PC2 computer calculated the optical rotation of the sample using Eq. (12). In Table 1 are the results concerning three crystals of BSO. As validation of the results obtained, the method of the polarimeter was implemented (see Table 1) [15]. These results show that with crystals of the same family, the rotary power is not equal. The reason in this case is that the samples are doped with different impurities [12, 13]. The three text crystals $\left(\mathrm{Bi}_{12} \mathrm{SiO}_{20}\right)$ used have the same crystallographic configuration, with their face parallel to the crystallographic planes (110), (110), (100). The first face has an area of $10 \times 10 \mathrm{~mm}^{2}$, being the face by which the light is incident. The refractive index is 2.53 and the absorption coefficient $-68 \mathrm{~m}^{-1}$; these values correspond to the wavelength of the laser used in the experiment $(\lambda=633 \mathrm{~nm})$.

In conclusion, the idea of this work is to show the operation of a new technique for measuring optical activity. Optimizing the technique to determine its accuracy, advantages and disadvantages, among other technical aspects, are the issues that will be discussed in future work. Furthermore, the system enables dynamic variations that follow the optical rotation, by only observing the intensity of harmonics.

Due to experimental conditions of our experiment, we do not treat the contrast of the fringes in terms of temporal coherence [16]. However, it is known that depending on the experimental conditions (coherence of the source, sample type and the type of optical arrangement) may be necessary to include the correct term modulation of the fringe pattern. We used a He-Ne laser of $633 \mathrm{~nm}$ medium wavelength and coherence length $\mathrm{L}_{\mathrm{c}} \approx 400 \mathrm{~m}$ and an amplitude division interferometer. The test crystals $6 \mathrm{~mm}$, $4 \mathrm{~mm}$ and $2 \mathrm{~mm}$ thick introduce an additional optical path difference of $15.18 \mathrm{~mm}, 10.12 \mathrm{~mm}$ and $5.06 \mathrm{~mm}$, respectively, for a refractive index value of 2.53 (for $633 \mathrm{~nm}$ wavelength). In our experiment the effect on the visibility of the fringes due to this additional path difference introduced by each sample (BSO) is of the form $V \approx \exp \left\{-(n-1) e / L_{c}\right\}$. If we made a quick calculation, we could prove that the temporal coherence in our experiment does not contribute to visibility deterioration of the fringe pattern when samples are introduced. In this sense, we have guaranteed that the change in the contrast of the interference pattern was generated only by the optical activity of each test crystal used.

\section{References}

[1] L. A. Nafie, Vibrational Optical Activity: Principles and Applications (Noida, John Wiley \& Sons Ltd 2011).

[2] W. Yang, P.R. Griffiths, G.J. Kemeny, Appl. Spectr. 38, 37 (1984).

[3] L.P. Polavarapu, Vibrational spectra: principles and applications with emphasis on optical activity (Amsterdam, Elsevier 1998).

[4] L. Barron, Molecular light scattering and optical activity (New York, Cambridge Univ. Press 2004).

[5] D. Tsankov, A. Polyanichko, H. Wieser,Vibrational Circular Dichroism: Ensuring Quality of Pharmaceutical Products. Pharmaceutical Sciences Encyclopedia (2011).

[6] S. Zhang, Z. Tian, Q. Wang, X. Yin, S. Li, Opt. Laser Techn. 39, 507 (2007).

[7] E. Shamonina V.P. Kamenov, K.H. Ringhofer, G. Cedilnik, A. Kießling, R. Kowarschik, D.J. Webb, Opt. Comm. 146, 62 (1998).

[8] V. Marinova, Opt. Mat. 15, 149 (2000).

[9] R.M. Ribeiro, A.B.A. Fiasca, P.A.M. dos Santos, M.R.B. Andreeta, A.C. Hernandes, Opt. Mat. 10, 201 (1998).

[10] D.G. Papazoglou A.G. Apostolidis, E.D. Vanidhis, App. Phys. B 65, 499 (1997).

[11] X. He, H. Zhou, W. Zhou, Z. Hu, C. Zhang, H. Huo, J. Wang, Y. Zuo, F. Lu, J. Cryst. Growth 351, 182 (2012).

[12] V. Tassev, G. Diankov, M. Gospodinov, Mat. Research Bulletin 30, (10), 1263 (1995).

[13] V. Tassev, M. Gospodinov, M. Veleva, Opt. Mat. 13, 249 (1999).

[14] J. E. Rueda-P, A. L. Becerra, Phot. Lett. Poland, 2(4), 174 (2010).

[15] L.A. Guerra, J.E. Rueda, Master's thesis in Physics: Dynamic Holography using photorefractive materials $\mathrm{Bi}_{12} \mathrm{SiO}_{20}$ and $\mathrm{LiNbO}_{3}$ (Universidad de Pamplona, Colombia, 2011)

[16] E. Hecht, Optics, Chapter 12. Basics of coherence theory (Addison Weley, 2002). 